\title{
Dynamics of the interaction of dust particles with the LHC beam
}

\author{
B. Lindstrom $\odot,{ }^{1,2}$ P. Bélanger $\odot,{ }^{1,3}$ A. Gorzawski $\odot,{ }^{1,4}$ J. Kral, ${ }^{1,5}$ A. Lechner, ${ }^{1}$ B. Salvachua $\odot,{ }^{1}$ \\ R. Schmidt, ${ }^{1}$ A. Siemko, ${ }^{1}$ M. Vaananen, ${ }^{1}$ D. Valuch $\odot,{ }^{1}$ C. Wiesner, ${ }^{1}$ \\ D. Wollmann $\oplus^{1}$, and C. Zamantzas $\odot^{1}$ \\ ${ }^{1}$ CERN, 1211 Geneva 23, Switzerland \\ ${ }^{2}$ Uppsala University, Department of Physics and Astronomy, Box 516, 75120 Uppsala, Sweden \\ ${ }^{3}$ TRIUMF, 4004 Wesbrook Mall, Vancouver, BC, V6T 2A3, Canada \\ ${ }^{4}$ University of Malta, Msida, MSD 2080 Malta \\ ${ }^{5}$ Faculty of Nuclear Sciences and Physical Engineering, Czech Technical University in Prague, 11519 \\ Prague, Czech Republic
}

\author{
(Received 8 November 2020; accepted 14 December 2020; published 30 December 2020)
}

\begin{abstract}
Micrometer-sized dust particles present in the Large Hadron Collider (LHC) beam pipe are believed to have caused many thousands of sporadic beam loss events around the LHC. These so-called unidentified falling objects (UFOs) have been under continuous study since the start of high intensity beam operation in the LHC due to their impact on the LHC availability: 139 beam dumps and 12 magnet quenches during Run II (2015-2018) alone. To mitigate the impact of UFOs on future accelerators such as the High Luminosity LHC and the Future Circular Collider, it is fundamental to foster a better understanding of these beam loss events. In this paper, key observations made since the start of LHC operation are summarized and the prevailing UFO hypothesis is confronted by a compilation of observations acquired during Run II. In particular, it is shown that UFOs must carry an initial negative charge before entering the proton beam, or that they are by some other means accelerated toward the beam not only by gravity. A simulation model for the dynamics of the dust particles and their interaction with the beam was developed over the years. This model is improved and validated by measurements. It is however also shown that a subset of observed beam losses, which contain a positive time profile skewness, cannot be explained by it.
\end{abstract}

DOI: $10.1103 /$ PhysRevAccelBeams.23.124501

\section{INTRODUCTION}

Spurious beam loss spikes distributed throughout the LHC have been present ever since high intensity beam operations began [1]. These events, believed to be caused by micrometer-sized dust particles, came to be known as unidentified falling objects (UFOs). They can cause beam losses intense enough to trigger beam dumps and magnet quenches [2], and they have had a detrimental effect on the machine availability in the LHC [3]. While the overall UFO rate has decreased over the years, it has been observed to increase after longer machine stops [4].

Similar dust-induced beam losses have occurred in electron and antiproton storage rings, where positively ionized dust was trapped in the beam core [5-7]. In the PF-AR (KEK) dust particles have been visually observed

Published by the American Physical Society under the terms of the Creative Commons Attribution 4.0 International license. Further distribution of this work must maintain attribution to the author(s) and the published article's title, journal citation, and DOI. Funded by Bibsam. to interact with the beam [8], and are thought to have been produced by electric discharges in a stripline beam position monitor [9]. Additionally, SuperKEKB suffered from sudden pressure bursts during beam commissioning thought to be caused by beam-dust interactions [10].

Several questions about their nature in proton machines remain, such as whether or not they can have an initial negative charge, what their source is, how they are released, why their rate changes over time, and most importantly, how to prevent them. Validation of the theoretical model is done through a simulation tool [11], which has been improved and benchmarked against beam loss measurements. This allows for a better understanding of their dynamics, which is key to answering the above questions.

In this paper, the different types of UFOs that have been observed are discussed. This is followed by an explanation of the current hypothesis, which is then tested using the UFO dynamics simulation tool together with beam loss measurements of overall time profiles, as well as losses arising from individual bunches. The latter allows calculating the dust particle position in relation to the beam center on a turn-by-turn basis. 


\section{UFO TYPES}

Throughout the LHC Run I (2009-2013) and Run II (2015-2018), specific locations around the LHC had an increased UFO activity leading to recurrent beam dumps. Studies were carried out in each case to mitigate the problem. A summary of the observations for all these specific cases are presented here. Their characteristic beam loss time profiles are plotted in Fig. 1.

\section{A. Standard UFO beam losses}

Figure 1(a) shows a standard UFO. They appear as sporadic beam loss spikes occurring throughout the whole accelerator, and their loss time profiles tend to have an approximately Gaussian shape, with a maximum duration of a few milliseconds. During Run II, 41 beam dumps and 8 magnet quenches were caused by standard UFOs. Each event causes a loss of up to $10^{8}$ protons from the beam.

\section{B. Injection kicker magnet UFOs}

UFOs at the injection kicker magnets (MKIs) had a significant impact on machine availability in 2010 and 2011, causing 35 beam dumps [12]. Unlike standard UFOs, which are present throughout the beam cycle, MKI UFOs typically occurred within 30 minutes after the last injection [13]. A typical beam loss time profile is shown in Fig. 1(b). There are no observable differences between these and the standard UFOs, aside from the location in the accelerator. Two dedicated experiments were carried out in 2011 and 2012 to study the release mechanism of MKI UFOs $[14,15]$. A clear correlation between pulsing the MKIs

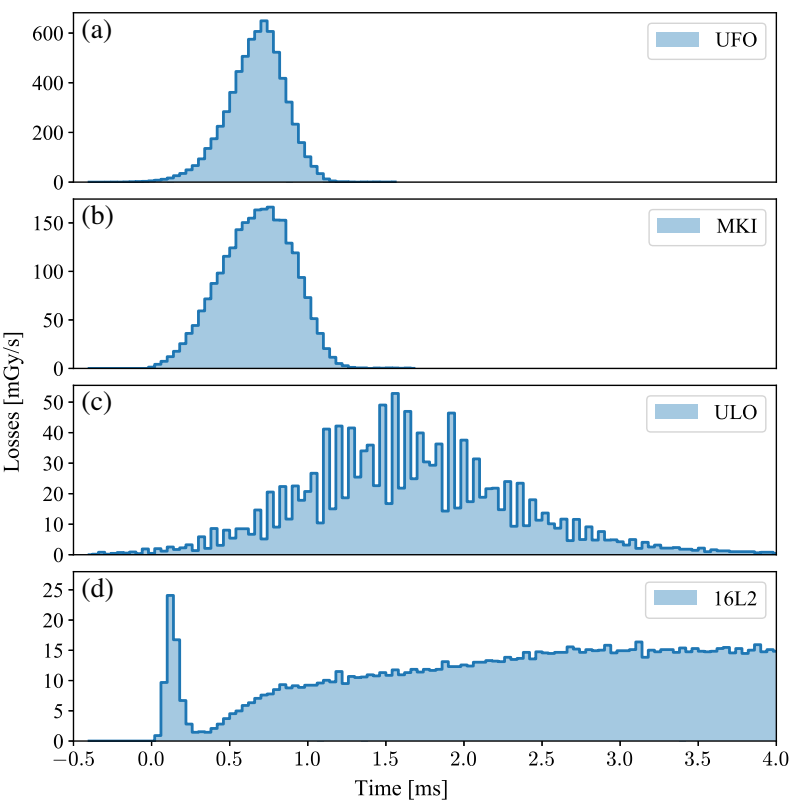

FIG. 1. Comparison of the loss signature of standard UFO losses (a) with three distinctive UFO types, MKI (b), ULO (c) and 16L2 (d), observed during Run II. and the occurrence of MKI UFOs was found. Following this observation, an improvement of the UFO detection system was implemented.

From FLUKA ${ }^{1}$ [16-18] simulations, it was shown that the UFOs had to be located in the MKIs in order to explain the observed loss pattern in the beam loss monitors (BLMs) surrounding them [13].

MKI UFOs were observed at the earliest $2 \mathrm{~ms}$ after beam injection. This delay is too short to be explained by gravity alone and it was suggested that $\mathrm{Al}_{2} \mathrm{O}_{3}$ particles from the ceramic tube of the MKIs could be charged by electron clouds and released due to vibrations or by the electric field of the MKI pulse [13], which is about $10 \mu \mathrm{s}$ long [19]. Based on the rate of inelastic collisions, it was also calculated that the minimum $\mathrm{Al}_{2} \mathrm{O}_{3}$ particle size required for the largest MKI UFO event was $45 \mu \mathrm{m}$. This is consistent with the dust inspection done on an MKI, removed from the accelerator in 2010, where more than five million particles with radii up to $100 \mu \mathrm{m}$ were found in a full MKI tank [12].

\section{Unidentified lying object}

In 2015, a high UFO activity was observed in a particular half-cell, leading to 14 beam dumps and 3 magnet quenches. Aperture measurements [20] revealed the presence of an object, so-called unidentified lying object (ULO), at the bottom of the beam pipe. It became apparent that the interaction of this object with the beam triggered UFO beam losses. As can be seen in Fig. 1(c), the losses from ULO events show a clear oscillation with a period of about $89 \mu \mathrm{s}$, corresponding to one LHC turn (each bin is $40 \mu \mathrm{s})$. This oscillating component is due to the fact that most ULO events were recorded with only a few bunches circulating in the LHC, leading to losses at one point in time for each LHC turn, followed by a long gap with no losses. To operate with a large number of bunches, a local orbit bump (horizontally and vertically) was implemented, bypassing the ULO. This drastically reduced the rate of observed UFO beam losses at the ULO and solved the problem. In 2019, the ULO was removed and identified as a strip of plastic originating from the beam pipe wrapping and introduced during the installation [4].

\section{16L2 type UFO}

In 2017, a new type of beam losses located near the quadrupole of the half-cell designated 16L2 led to 68 beam dumps and 1 magnet quench, compared to a total of about 300 beam dumps due to faults during the same period [21]. In 2018, 16 additional beam dumps were recorded. The typical signal for 16L2 events starts with a UFO-like spike followed by a fast loss rise [see Fig. 1(d)]. Many of the

${ }^{1}$ FLUKA is a Monte-Carlo simulation package for particle transport and their interaction with matter. 
beam dumps were triggered by BLMs in the betatron collimation region [22], where the losses increased continuously over several milliseconds. In-depth analysis of the losses revealed that the initial spike was due to the LHC bunches interacting with UFO-like solid matter, while the following beam losses were caused by transverse beam instabilities [23]. The probable cause for the losses observed in $16 \mathrm{~L} 2$ was the presence of solid nitrogen, oxygen, and water in the surrounding vacuum chamber, introduced by an accidental air inflow [21]. It is believed that solid particles of the contaminants sublimated to the gaseous phase following the interaction with the beam causing a fast beam instability [21,24]. Electron clouds were observed to increase the rate of events. The recurring beam dumps were consequently mitigated by reducing the multipacting, through different filling schemes, lower bunch intensities and additional magnetic fields [23]. The affected sector was later warmed up to $\sim 80 \mathrm{~K}$ to remove the frozen gas from the beam pipe. This improved the situation, but did not solve it, and in 2019 the sector was warmed up to room temperature.

\section{THE UFO HYPOTHESIS}

The prevailing UFO hypothesis is that macroscopic dust particles enter the beam producing beam losses due to their interaction with the protons. Previous work on the subject has shown consistency between the theoretical model and observations in several aspects: comparable beam loss time profiles [25], comparable distribution of the beam loss peaks during Run I [11] and consistency with dust contamination in the beam pipe [26]. The sequence of events goes as follows: (1) A dust particle falls from the beam screen toward the beam. (2) The dust particle is ionized due to collisions with the beam protons, releasing free electrons. (3) The now positively charged dust particle is repelled from the beam by its electric field. It is generally assumed that the dust particles are neutral prior to their interaction with the beam, implying that gravity is the sole force initially acting on them. The main observable for UFOs are the induced beam losses, and other plausible explanations for the observed beam losses have been rejected, leaving the dust particle hypothesis [13].

Studies have been carried out to inspect the dust present in the beam pipe of the LHC $[12,26]$. Dust containing the following elements were found: $\mathrm{Ca}, \mathrm{Ti}, \mathrm{Au}, \mathrm{In}, \mathrm{Al}, \mathrm{Si}, \mathrm{Ag}$, Fe. The particle radii ranged from $1 \mu \mathrm{m}$ to $200 \mu \mathrm{m}$. Small particles were predominant, with more than $90 \%$ of them having a radius smaller than $5 \mu \mathrm{m}$. More than half of the particles larger than this still has a radius smaller than $30 \mu \mathrm{m}$. Using this information as a plausible assumption for the initial conditions of UFOs, Monte-Carlo simulations showed [11] that the UFO hypothesis could be used to reproduce the distribution of beam loss peaks measured during Run I accurately. However, the simulated loss signature does not agree with all measured UFO events, in particular not the rise time of the beam losses.

\section{DATA COLLECTION}

UFO events create local beam losses due to inelastic collisions between the beam protons and the dust particle nuclei, as well as losses in the collimation regions due to scattering of protons with small angles. The losses have been recorded by two different beam loss monitoring systems during Run II, ionization chamber BLMs (ICBLMs) and diamond BLMs (dBLMs). The ICBLM system consists of about 3700 detectors distributed along the accelerator, measuring local beam losses. ICBLM signals are continuously monitored, and a recording with a $80 \mu$ s temporal resolution is triggered when UFOs are detected. This resolution corresponds to roughly one LHC turn $(89 \mu \mathrm{s})$. The ICBLMs are also recorded, with their maximum resolution of $40 \mu \mathrm{s}$, by the post-mortem system $[27,28]$ for every beam dump. All ICBLMs are calibrated individually using a gamma source $[29,30]$.

The dBLMs used for UFO studies consist of six units, with two per beam installed downstream of the primary collimators in the betatron collimation region, and one per beam installed around the 16L2 interconnect. The dBLM signal shaping and sample rate of $650 \mathrm{MHz}$ are fast enough to distinguish losses due to individual bunches in the beam [31,32], given the bunch spacing of $25 \mathrm{~ns}$. The readout is triggered at every beam dump. In addition, a real-time UFO detection method running within the readout system is used to trigger the readout during beam operation. The method analyzes the beam losses on-the-fly, and triggers high precision data readout when a suspected beam loss shape is detected. In these studies, the dBLMs are cross-calibrated to the ICBLMs, since the latter have a larger volume and are more precise.

In total 337,217 ICBLM data dumps were triggered during Run II. For 57,262 of these, ICBLM time profiles were successfully recorded. On these, filters were applied to ensure a sufficient signal-to-noise ratio and a minimum signal length of $5 \times 80 \mu \mathrm{s}$. With these filters the number of studied events was narrowed down to 2,964. The filters were chosen to identify the time profiles on which it is possible to make meaningful numerical analysis. All validated UFO events are summarized in Table I.

TABLE I. Validated UFO events collected during Run II. The ICBLM measurements are a subset of validated measurements as detailed in the text.

\begin{tabular}{lcrcrr}
\hline \hline Detector & Resolution & UFO & MKI & ULO & 16L2 \\
\hline dBLM & $1.54 \mathrm{~ns}$ & 37 & 0 & 0 & 54 \\
ICBLM & $40 \mu \mathrm{s}$ & 47 & 0 & 14 & 84 \\
ICBLM & $80 \mu \mathrm{s}$ & 2958 & 6 & 0 & 0 \\
\hline \hline
\end{tabular}




\section{SKEWED GAUSSIAN SIGNATURE}

Previous work mentioned a time profile asymmetry of the UFO losses [2,13]. Data collected during both Run I and Run II showed asymmetric Gaussian time profiles with about half the UFO events having a signal with longer rise time (negatively skewed) and half having a longer fall time (positively skewed). Measurement examples of a positively skewed and a negatively skewed UFO loss profile are shown in Fig. 2. For a constant dust particle velocity, the inelastic collision rate follows the beam profile (Gaussian) as the dust is passing through. However, due to the interaction with the proton beam, the dust is ionized, leading to an asymmetry in the incoming and outgoing speed of the dust particle.

The observation of positively skewed events is inconsistent with the current UFO hypothesis explained in Sec. III, which can only account for negatively skewed time profiles. As determined from the dynamics simulation tool explained in Sec. VI, which is based on this hypothesis, dust particles generally acquire a positive charge between $10^{6} \mathrm{e}$ and $10^{11} \mathrm{e}$ e is the elementary charge, approximately $1.602 \times 10^{-19} \mathrm{C}$. Given the electric field of the LHC beam with nominal parameters (2808 bunches of $1.15 \times 10^{11} \mathrm{p}^{+}$) and a UFO charge of $10^{9} \mathrm{e}$, the acceleration is on the order of $10 \mathrm{~km} / \mathrm{s}^{2}$ a few $\sigma$ away from the beam. For a UFO event entering and leaving the beam at $6 \sigma$, with a turning point around $2 \sigma$, one can calculate that the entry speed of a neutral dust particle is around $0.5 \mathrm{~m} / \mathrm{s}$ due to gravity, while the exit speed is around $5 \mathrm{~m} / \mathrm{s}$, leading to a negatively skewed loss signal. In this section a unimodal skewed distribution is proposed to quantify this asymmetry and to study the discrepancy between the model and the experimental observations.

A unimodal skewed distribution is a distribution showing a single highest value with a left-right asymmetry, having a longer tail on one side. In particular, the skew normal distribution is defined as follows
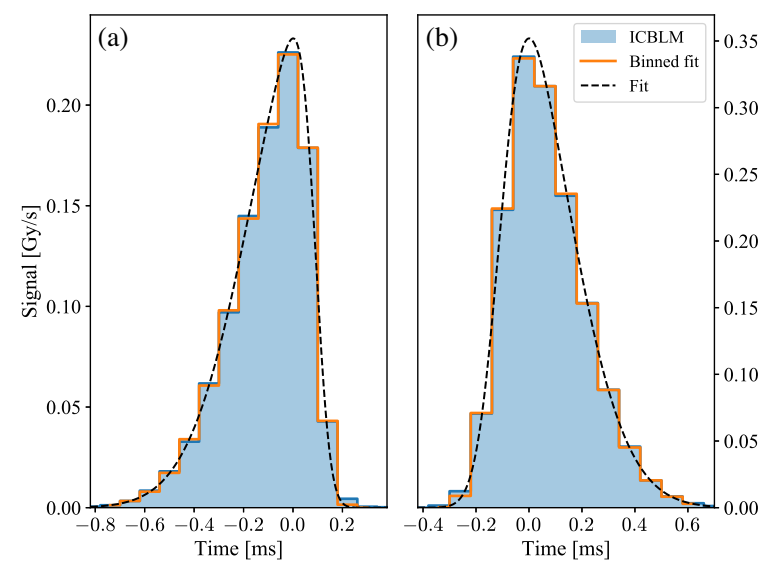

FIG. 2. Example of measured UFO events with negative skewness (a) and positive skewness (b). The fit for the underlying inelastic collision rate is shown, as well as the resulting binned signal. Their skewness $\gamma$ is -0.87 and 0.68 respectively.

$$
\Phi(t)=A e^{-\frac{(t-\mu)^{2}}{2 \omega^{2}}}\left(1+\operatorname{erf}\left[\frac{\alpha(t-\mu)}{\sqrt{2 \omega^{2}}}\right]\right),
$$

where $A, \omega, \mu$, and $\alpha$ are the amplitude, scale, location, and shape parameters, respectively. The shape parameter is linked to the skewness of the distribution (the third standardized moment), $\gamma_{1}$ through:

$\gamma_{1}=\frac{4-\pi}{2}\left(\frac{\delta}{\sqrt{\pi / 2-\delta^{2}}}\right)^{3}$ with $\quad \delta=\frac{\alpha}{\sqrt{1+\alpha^{2}}}$.

Apart from the events that triggered beam dumps, the beam losses are measured by ICBLMs and integrated in $80 \mu$ s bins. For this reason, Eq. (1) is binned in the same way as the measurements and a nonlinear least squares method is applied to find the best fit on the binned data. Good fits were obtained for both positively and negatively skewed events, as shown in Fig. 2. A UFO signal can, thus, be uniquely described by three distribution parameters, the location parameter being arbitrary. The amplitude parameter, $A$, can be used to describe the magnitude of the losses in Gy/s or in terms of the inelastic collision rate using conversion factors determined through FLUKA simulations [33]. The parameter $\omega$ scales the width of the function and provides a measure of the time that the UFO spent interacting with the beam. The shape parameter $\alpha$ can be used to compute the skewness $\gamma_{1}$ following Eq. (2).

The time profile asymmetry of UFO losses was observed in Run I and briefly discussed [[13] Fig. 5.9, p. 68]. The equivalent measured asymmetry for Run II events is presented here in Fig. 3. Note that the plot in the reference shows an estimate of the negative $\gamma_{1}$. In both cases, there is

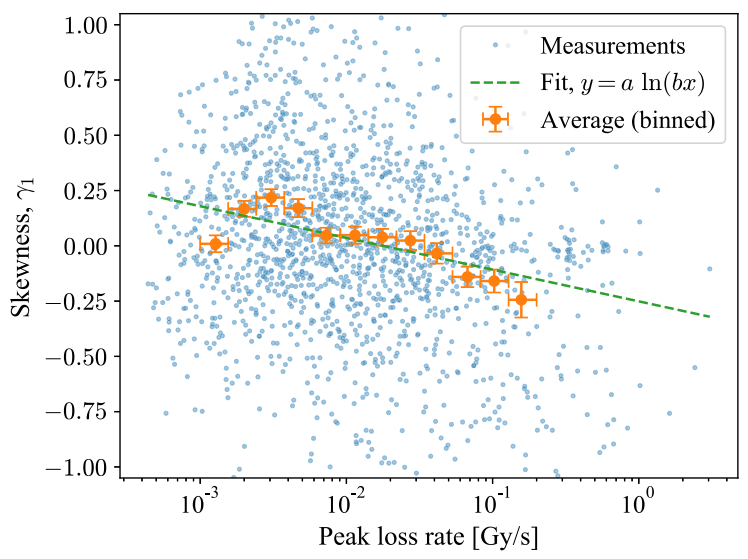

FIG. 3. Measured skewness and peak loss rate of 1719 UFO events observed during Run II. The fit parameters are $a=$ $-0.062 \pm 0.007$ and $b=56 \pm 11$. Only events with sufficient signal which occurred for colliding beams at top energy are shown. The orange dots indicate the average skewness and average peak signal of the data within the bins defined by the horizontal bars and standard error of the mean shown by vertical bars. 
a slight decrease of the average skewness as a function of the peak loss rate. The distribution observed during Run II is similar to the one observed during Run I.

\section{SIMULATING UFO DYNAMICS}

By simulating the dynamics of the dust particles under the influence of the beam potential it is possible to determine the resulting beam losses and how they depend on the UFO properties (mass, material, size), initial conditions (charge, position, velocity) and beam properties (energy, intensity, transverse size). The simulated losses can then be compared to the ICBLM and dBLM measurements.

A UFO dynamics simulation tool was consequently developed, following the hypothesis explained in Sec III. This work has been ongoing since 2010 [11,25,34]. It works by numerically solving the equations of motion given the forces due to the beam electric field and gravity. As it interacts with the beam, it calculates the rate of ionization due to escaping secondary electrons, as well as the produced beam losses from inelastic collisions with the dust particle nuclei. The code was translated to PYTHON and updated once more, as described below. The reader should refer to [11] for the most complete description of the model prior to the latest updates presented here.

\section{A. Electric field of the beam}

Prior to this paper, the impact of the beam screen on the electric field of the beam was either neglected, or approximated by adding a contribution from image charges coming from the top and bottom horizontal surfaces of the beam screen only. This description is only valid in the immediate vicinity of the beam.

The method used to efficiently compute the total electric field with the appropriate boundary condition at the beam screen is described in detail in [35]. One can place a series of $N$ infinitely long lines of image charges around the outer surface of the beam screen with linear charge densities $\lambda_{i}$. Applying the Laplace equation then yields a system of $N$ equations to find the values of $\lambda_{i}$ :

$$
\begin{aligned}
& -\frac{1}{2 \pi \varepsilon_{0}}\left[\begin{array}{ccc}
\ln \left[\left|\vec{r}_{1} / k-\vec{r}_{1}\right|\right] & \ln \left[\left|\vec{r}_{1} / k-\vec{r}_{2}\right|\right] & \ldots \\
\ln \left[\left|\vec{r}_{2} / k-\vec{r}_{1}\right|\right] & \ln \left[\left|\vec{r}_{2} / k-\vec{r}_{2}\right|\right] & \ldots \\
\vdots & \vdots & \ddots
\end{array}\right]\left[\begin{array}{c}
\lambda_{1} \\
\lambda_{2} \\
\vdots
\end{array}\right] \\
& =\frac{\lambda_{B}}{2 \pi \varepsilon_{0}}\left[\begin{array}{c}
\ln \left[\left|\vec{r}_{1} / k-\vec{r}_{B}\right|\right] \\
\ln \left[\left|\vec{r}_{2} / k-\vec{r}_{B}\right|\right] \\
\vdots
\end{array}\right],
\end{aligned}
$$

where $\vec{r}_{B}$ is the position of the center of the beam in the beam screen and $k=\left|\vec{r}_{i}\right| /\left|\vec{r}_{\text {screen }}\right|$ describes how far from the beam screen the line of image charges is placed, so that $\vec{r}_{i} / k$ is the position of the beam screen right under the $i$ th line of image charges. $\lambda_{B}=N_{p} / C$ is the linear charge density of the LHC beam, where $N_{p}$ is the number of protons in the beam and $C$ is the circumference of the LHC. The $\lambda_{i}$ are found by using the inverse matrix method. Neglecting the contribution from this distribution of image charges leads to field errors of more than $10 \%$ for locations $30 \sigma$ away from the beam, and $1 \%$ at $10 \sigma$. In order to simulate the dynamics of initially charged UFOs, it is necessary to have an accurate description of the electric field everywhere around the beam since it has a considerable impact on the speed and the trajectory of the dust particle during its approach.

\section{B. Escaping knock-on electrons}

Correctly describing the charging rate of UFOs interacting with the beam is key to correctly simulating their dynamics. The calculation of the average number of escaping electrons per passing proton in the dust particle was reviewed and compared to FLUKA.

In the model, UFOs are charged from escaping knock-on electrons created by the passage of high energy protons in the dust particle. The energy and angular distribution for energetic knock-on electrons are given in [36]. To compute the UFO charging rate, the distribution of secondary electrons with sufficient energy to escape the dust particle is integrated. The minimum energy required for a UFO of radius $R$ is

$$
T_{\min }=\frac{Q e}{4 \pi \varepsilon_{0} R}+W
$$

where $Q$ is the charge and $W$ is the work function of the dust particle. The first term of Eq. (4) is the averaged Coulomb potential inside a uniformly charged sphere. To find the work function of high energy electrons, the calculation from previous authors [11] is used with small adjustments. Following the empirical relation described in [37] for $0.3 \mathrm{keV}$ to $20 \mathrm{MeV}$ electrons, the practical range $L(T)$ of electrons in matter is given by:

$$
L(T)=\frac{A T}{\rho}\left(1-\frac{B}{1+C T}\right)
$$

where $A=5.37 \times 10^{-6} \mathrm{~kg} \mathrm{~m}^{-2} \mathrm{eV}^{-1}, B=0.9815, C=$ $3.123 \times 10^{-6} \mathrm{eV}^{-1}$ and $\rho$ is the density of the material.

The work function of the dust particle is found by equating the practical range to the average transverse path length an electron created inside the dust particle has to travel in order to reach the surface. Based on the FLUKA simulations, one finds $L(W)=0.3216 R$ such that $W=L^{-1}(0.3216 R)$.

Following these changes to the model, the average number of escaping electrons per passing proton for a neutral UFO agrees well with FLUKA, as shown in Fig. 4 for the materials copper and carbon. The spectrum of the electron kinetic energy as they exit the dust particle also 


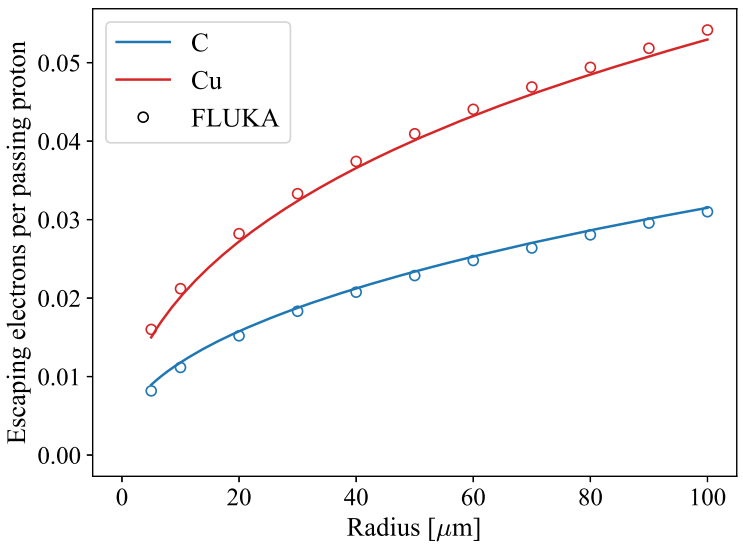

FIG. 4. Average number of escaping electrons per passing proton for a neutral UFO. The updated model (solid lines) is compared to FLUKA (circles).

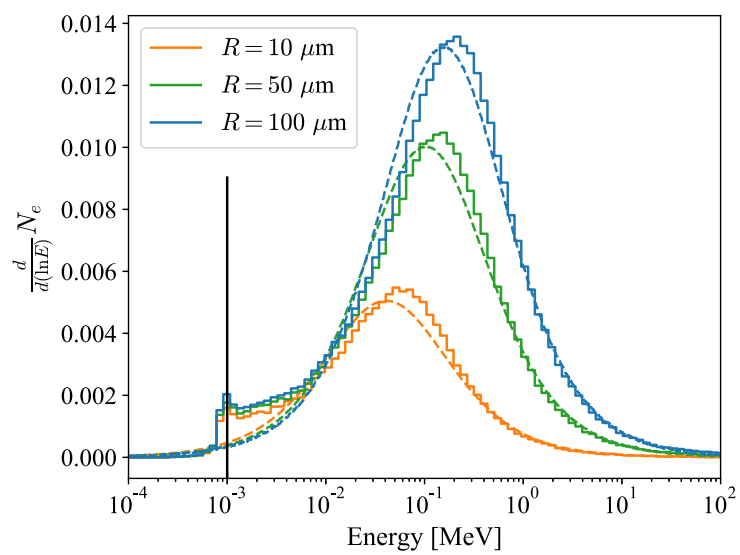

FIG. 5. Energy spectrum of knock-on electrons as they escape the UFO. The updated model (dashed lines) is compared to FLUKA (solid lines) for three dust particle radii. The energy cut for electron transport in FLUKA is shown by the black line, at $1 \mathrm{keV}$.

agrees well with FLUKA, as shown in Fig. 5. Note that FLUKA is a condensed-history transport code and therefore cannot simulate low-energy $(\lesssim 1 \mathrm{keV})$ electron emission from the dust particle. Hence the contribution of lower-energy electrons to the overall charging rate is not accounted for, indicated by the black vertical line in the figure.

These changes do not drastically change the global distribution of simulated losses obtained when simulating UFOs with varying parameters. However, since the charging rate influences the penetration depth of a given UFO, the specific UFO parameters leading to a precise loss profile can be significantly modified.

\section{INITIAL CHARGE}

To test the hypothesis that the UFO dust particles are initially neutral $\left(Q_{0}=0\right)$, Monte-Carlo simulations of
TABLE II. Input parameters for Monte-Carlo simulations.

\begin{tabular}{lc}
\hline \hline Parameter & Values \\
\hline UFO $Q_{0} / m$ & $-10^{-8}--10^{0} \mathrm{C} / \mathrm{kg}$ \\
UFO Position & Top of beam screen \\
UFO Radius & $1 \mu \mathrm{m}-100 \mu \mathrm{m}$ \\
UFO Material & $\mathrm{C}, \mathrm{Cu}, \mathrm{Si}, \mathrm{Al}$ \\
Beam Energy & $6.5 \mathrm{TeV}$ \\
Beam Intensity & $3 \times 10^{11}-3 \times 10^{14}$ \\
Beam $\sigma_{x}$ and $\sigma_{y}$ & $80 \mu \mathrm{m}-260 \mu \mathrm{m}$ \\
\hline \hline
\end{tabular}

UFO dynamics were performed with the parameters found in Table II for an initially neutral and an initially negatively charged dust particle.

The rise time $R_{t}$ is defined as the time required for the BLM response to rise from $1 \%$ to $100 \%$ of its peak value. The resulting distributions for the rise time of the simulated loss signals obtained with the Monte-Carlo simulations are shown in Fig. 6 and compared to the distribution found in the ICBLM measurements. Plot (a) shows the simulated distribution for initially neutral dust particles, whereas plot (b) shows the resulting distribution when allowing the initial charge-to-mass ratio to vary. The vertical black line indicates the fastest rise time for neutral dust particles.

Even after excluding the shortest UFO events (with less than 5 data points), it is found that $40 \%$ of Run II events have rise times too short to be explained by an initially neutral dust particle, for which $R_{t}>262 \mu \mathrm{s}$ based on the simulations. However, when considering dust with negative charge-to-mass ratios on the order of $10^{-3} \mathrm{C} / \mathrm{kg}$, the

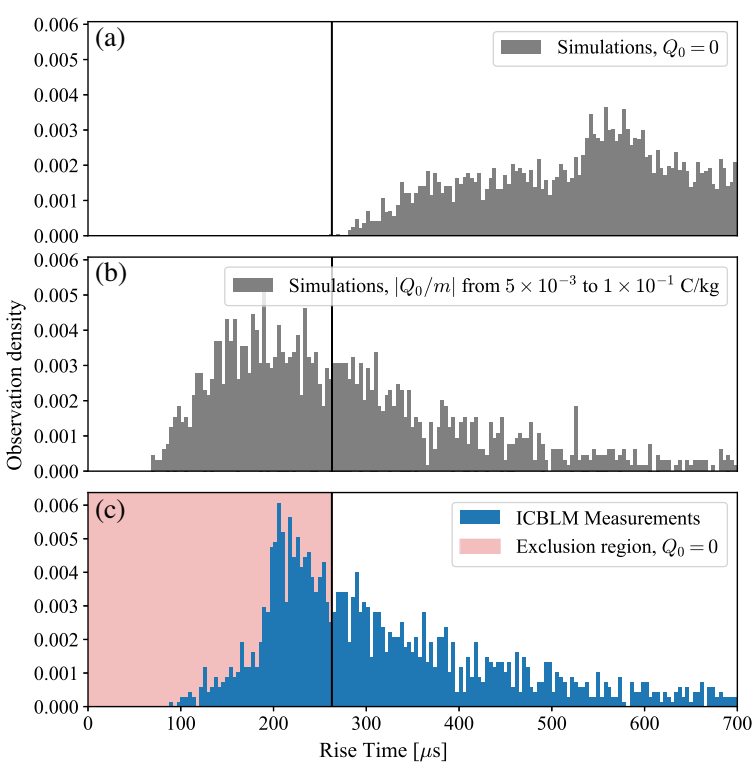

FIG. 6. Distribution of rise times $R_{t}$ found in Monte-Carlo simulations for initially neutral UFOs (a), for initially negatively charged UFOs (b) and found in ICBLM measurements for colliding beams at top energy (c). 
distribution of rise times is in good agreement with the measurements. This result strongly suggests that the dust particles in UFO events are initially negatively charged. This might also play a role in their release mechanism. Furthermore, the simulated charge-to-mass ratios are consistent with observations in cosmic dust, where the ratio for micrometer-sized particles is generally between $10^{-4}$ and $10^{-1} \mathrm{C} / \mathrm{kg}[38]$ in conditions similar to those in the LHC.

\section{UFO POSITION MEASUREMENT}

In 2017, a proof-of-principle study on the dynamics of UFOs was performed in the LHC [39]. In this experiment, selected bunches had their emittances blown-up, one group vertically and another group horizontally, meaning that they had wider proton distributions in the respective planes. A 16L2 type UFO was then triggered [23] and the resulting beam losses were measured by the dBLMs installed close-by. The method of the proof-of-principle study was applied in 2018 for the study of standard UFOs. These measurements are analyzed and discussed in the following sections.

\section{A. Ratio of losses method}

Beam losses are proportional to the proton flux through the dust particle, and the beam loss measurements thus constitute an indirect measurement of the proton density, for each bunch individually. The UFO position in relation to the beam center can be inferred by comparing the amplitude of the beam losses from three different bunch groups; vertically blown-up bunches, horizontally blownup bunches and reference bunches without any blow-up.

For example, a dust particle at a position vertically above the beam in the tails of the beam distribution sees a larger proton density in the vertically blown-up bunches than in the reference bunches. On the other hand, for a dust particle in the center of the beam, the reference bunches would provide a larger proton density at the position of the dust particle than the blown-up bunches.

Here follows an example. Three binormal bunch distributions are assumed: the reference bunch [shown in Fig. 7(c)], with values for $\sigma_{x}=0.28 \mathrm{~mm}$ and $\sigma_{y}=0.12 \mathrm{~mm}$, a bunch with the horizontal emittance blown-up ( $\sigma_{x}=$ $0.33 \mathrm{~mm}$ and $\sigma_{y}=0.13 \mathrm{~mm}$ ), and a bunch with the vertical emittance blown-up ( $\sigma_{x}=0.30 \mathrm{~mm}$ and $\sigma_{y}=0.16 \mathrm{~mm}$ ). It is to be noted that a blow-up in one plane leads to a small blow-up in the other plane.

In this example, the beam losses from the interaction of the dust particle with the horizontally and vertically blownup bunches is a factor of two above the beam losses of the reference bunches. By dividing the proton density of the vertically blown-up bunch by the proton density of the reference bunch, one obtains an elliptical contour line [Fig. 7(a)]. The dust particle must have been on this line in order to produce the measured losses. By dividing the

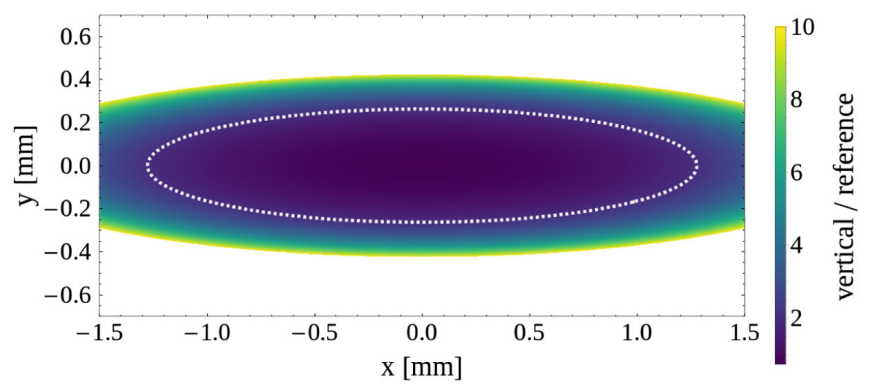

(a)

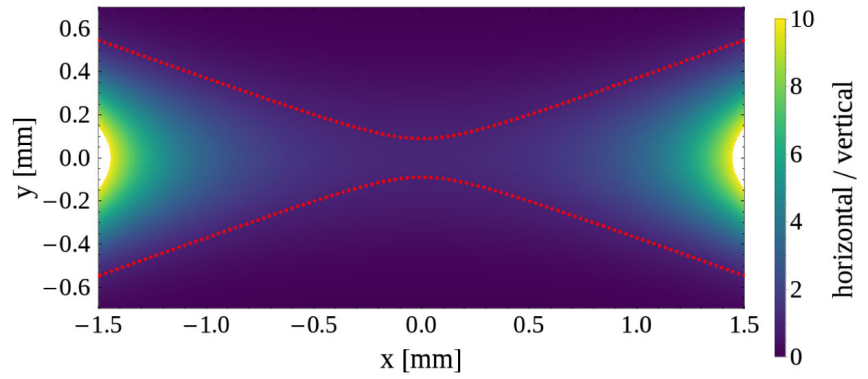

(b)

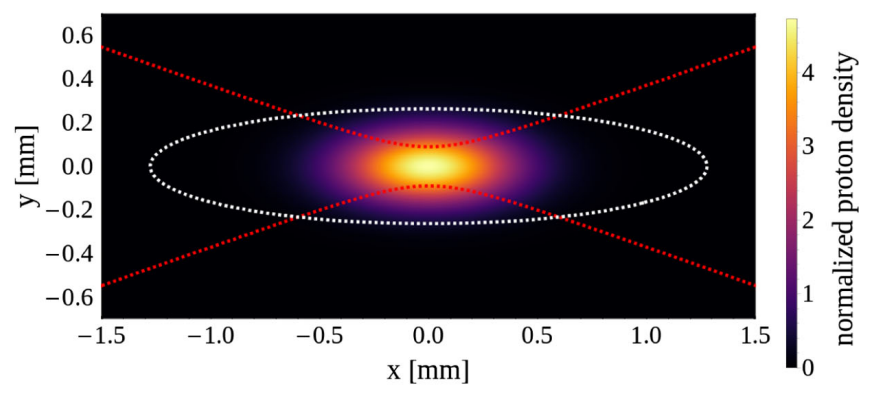

(c)

FIG. 7. (a) vertically blown-up bunch distribution divided by that of the reference bunches, (b) horizontally blown-up bunch distribution divided by that of the vertically blown-up bunch, (c) reference bunch distribution. The contour line in (a) corresponds to a factor of 2, whereas the contour lines in (b) corresponds to a factor of 1 . These contour lines are overlayed on the reference bunch distribution in (c).

proton density of the horizontally blown-up bunch by that of the vertically blown-up bunch, one obtains a hyperbolic contour line [Fig. 7(b)]. One finds four intersection points between the two contour lines [Fig. 7(c)]. These four points, distributed symmetrically around the beam center, constitute the estimates of the dust particle position.

This method is contingent on the following: (i) the bunch intensity must be known and used to normalize the measured beam losses, (ii) the bunch distribution must be known, including the width (emittance, beta function, and dispersion), (iii) all bunches are assumed to be on the same orbit, (iv) beam losses are assumed to be identical, and be detected with the same efficiency, independently of the bunch shape, (v) the dust particle is assumed to move slowly enough between its interactions with the different 
types of bunches that it can be considered static, (vi) single bunch resolution in the beam loss measurements.

Among the listed requirements, in particular the bunch distribution has a certain uncertainty, since the online beam profile measurements in the LHC are not sensitive to the beam halo from around $4.5 \sigma$ and out [40-42]. Nevertheless, the small proton density in the halo has a comparatively small effect on the dust particles, since they tend to reach at least $3 \sigma$ from the bunch center at their closest approach. In this paper, binormal bunch distributions with an emittance as measured through the synchrotron radiation [43] are thus considered.

\section{B. Analytical approach}

The measured beam losses per bunch are, under the assumption of Gaussian beams, proportional to:

$$
\text { signal } \propto \frac{N_{b}}{\sigma_{x} \sigma_{y} \sqrt{2 \pi}} e^{-\frac{1}{2}\left(\frac{x^{2}}{\sigma_{x}^{2}}+\frac{y^{2}}{\sigma_{y}^{2}}\right)} .
$$

The proportionality factor depends on the amount of losses produced per collision, shower production and the detector efficiency. These factors are independent of which bunch the dust is interacting with. $N_{b}$ is the bunch intensity, $x$ and $y$ the horizontal and vertical coordinates of the dust particle center in relation to the bunch center, and $\sigma_{x}$ and $\sigma_{y}$ the transverse bunch sizes.

Defining $m_{i}$ as the measured losses normalized by bunch intensity for bunch type $i$, the ratio of the measurements from two bunch types $i$ and $j$ are given by:

$$
\begin{aligned}
\frac{m_{i}}{m_{j}} & =\frac{\sigma_{x j} \sigma_{y j} \exp \left[-\frac{1}{2}\left(\frac{x^{2}}{\sigma_{x i}^{2}}+\frac{y^{2}}{\sigma_{y i}^{2}}\right)\right]}{\sigma_{x i} \sigma_{y i} \exp \left[-\frac{1}{2}\left(\frac{x^{2}}{\sigma_{x j}^{2}}+\frac{y^{2}}{\sigma_{y j}^{2}}\right)\right]} \\
& =\frac{\sigma_{x j} \sigma_{y j}}{\sigma_{x i} \sigma_{y i}} \exp \left[\frac{1}{2}\left(x^{2}\left(s_{x j}^{2}-s_{x i}^{2}\right)+y^{2}\left(s_{y j}^{2}-s_{y i}^{2}\right)\right)\right],
\end{aligned}
$$

where the reciprocal of the bunch size was defined as $s_{x, y} \equiv 1 / \sigma_{x, y}$. Rearranging this gives an equation describing a hyperbola or an ellipse:

$m_{i j} \equiv 2 \ln \left[\frac{m_{i}}{m_{j}} \frac{\sigma_{x i} \sigma_{y i}}{\sigma_{x j} \sigma_{y j}}\right]=x^{2}\left(s_{x j}^{2}-s_{x i}^{2}\right)+y^{2}\left(s_{y j}^{2}-s_{y i}^{2}\right)$,

where $m_{i j}$ was defined for brevity. This equation can be solved uniquely with a four-fold symmetry by adding the ratio between a third bunch and one of the first two bunches.

\section{Error propagation}

Error propagation gives the covariance matrix for the estimated $\mathrm{x}$ and $\mathrm{y}$ coordinates from the covariance matrix of $m_{i j}$, that is $C_{X}=J C_{M} J^{T}$, where $J$ is the Jacobian for the transformation from the measured $m_{i j}$ to the $\mathrm{x}$ and $\mathrm{y}$ estimates, which is determined by solving Eq. (8). $C_{X}$ and $C_{M}$ are the covariance matrices for the respective parameters.

The sample standard deviation $\hat{\sigma}$ of the measurements can be estimated from bunches with similar bunch parameters, meaning that they should provide the same amount of beam losses given a set dust particle position. This should be the same for the blown-up bunches. The covariance matrix for the measured parameters, considering the three types of bunches (reference, vertically, and horizontally blown-up) is then given by:

$$
\left(\begin{array}{ccc}
\sigma_{1}^{2} & 0 & 0 \\
0 & \sigma_{2}^{2} & 0 \\
0 & 0 & \sigma_{3}^{2}
\end{array}\right)=\hat{\sigma}^{2}\left(\begin{array}{ccc}
\frac{1}{N_{1}} & 0 & 0 \\
0 & \frac{1}{N_{2}} & 0 \\
0 & 0 & \frac{1}{N_{3}}
\end{array}\right),
$$

where $N_{i}$ are the number of bunches of each type. The covariance matrix for $m_{i j}$ then transforms to the following:

$$
C_{M}=4 \hat{\sigma}^{2}\left(\begin{array}{ccc}
\frac{1}{N_{1} m_{1}^{2}}+\frac{1}{N_{2} m_{2}^{2}} & 0 & \frac{1}{N_{2} m_{2}^{2}} \\
0 & 0 & 0 \\
\frac{1}{N_{2} m_{2}^{2}} & 0 & \frac{1}{N_{2} m_{2}^{2}}+\frac{1}{N_{3} m_{3}^{2}}
\end{array}\right) .
$$

The zero values in this matrix correspond to the parameter $m_{22}$ and can thus be removed from the matrix, giving the required $2 \times 2$ matrix for calculating the final covariance matrix $C_{X}$. This is used to construct confidence ellipses for the estimated dust particle positions when applying the method on the measurements.

\section{UFO DETECTION}

Most UFOs do not provide beam losses intense enough to trigger beam dumps. Furthermore, due to the background losses in the collimation system, where the dBLMs are located, the losses from the UFOs are hidden, as an example shows in Fig. 8. The UFO signal is encircled in red.

In order to detect the UFOs within the background, two different triggering methods were utilized: (i) Number of peaks within a certain time window. This algorithm works as follows, it counts the number of peaks above a certain threshold (8200 bits) within three running $5 \mathrm{~ms}$ time windows. If the number of peaks in at least one LHC turn $(89 \mu \mathrm{s})$ in one time window is larger than 100 , and then less than 100 in all LHC turns within the following and preceding $5 \mathrm{~ms}$ time windows, the data is saved. (ii) Integration method. This method instead does a simple integration (summing all the sampled values) into $5 \mathrm{~ms}$ bins and if the value of one bin is above a certain threshold compared to the preceding and following bins, the data is saved. 


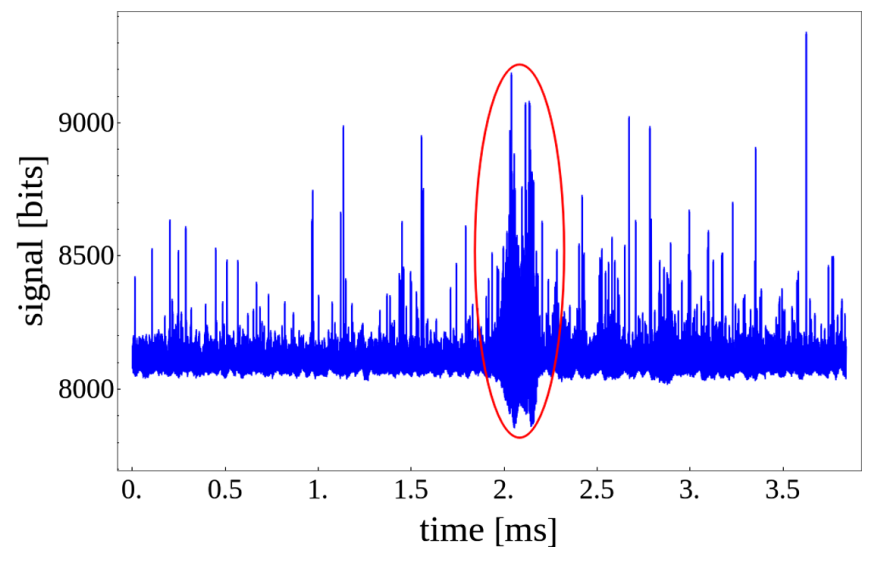

FIG. 8. Raw signal of a UFO recording by a dBLM downstream of the collimators. The UFO is encircled.

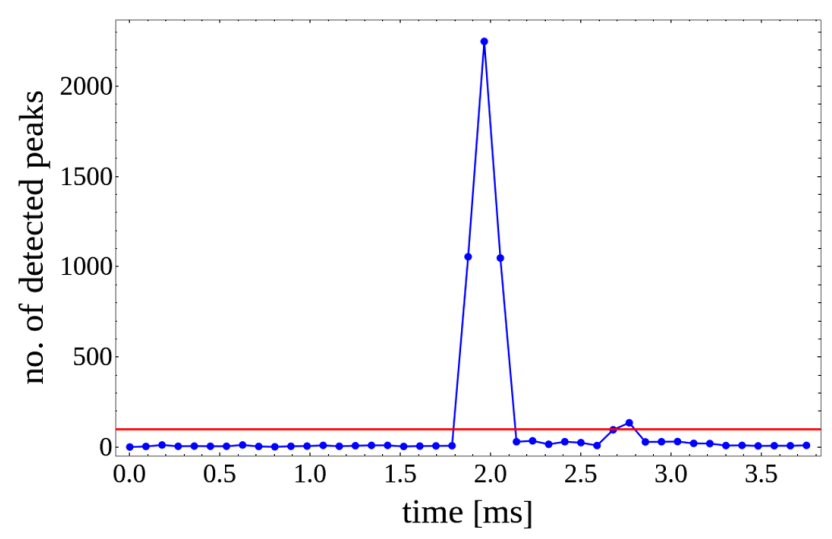

FIG. 9. Example of the peak counting algorithm, applied to the signal in Fig. 8. The red line shows the trigger threshold of 100 peaks per turn.

Due to a high volume and a long (three minutes) readout time of the raw dBLM data, the detection algorithm thresholds were tuned to neglect other sources of high beam losses as much as possible.

An example of the peak counting algorithm is shown in Fig. 9. For the background losses, the number of peaks per LHC turn remains around 10, whereas for the UFO the value 2247 is reached, meaning that most of the 2556 bunches present in the beam were detected. Due to an ac/dc converter in the readout system, some peaks fall below the cutoff at 8200 bits and are consequently not detected by the trigger system. However, all bunches interact with the UFO, providing losses, and can be analyzed in the postprocessing.

\section{EXPERIMENT WITH STANDARD UFOS}

An experiment involving blown-up bunches was prepared to study standard UFOs. Due to the low UFO rate of about two per beam day in 2018, the experiment had to be conducted in parallel to normal operation. At the beginning of the filling pattern for both beams, there is a train of twelve noncolliding bunches which could be utilized for the UFO studies. In both beams, two of these bunches were blown-up, one horizontally and one vertically.

\section{A. Beam setup}

Measured emittances of the 12 non-colliding bunches can be seen in Fig. 10 for beam 2 during a typical fill, before and after the energy ramp. The goal was to reach a factor of two larger emittance in the blown-up bunches, with one being blown-up vertically and one horizontally.

The blow-up was done during the injection of the beams. Consequently the horizontally blown-up bunches lost more intensity during energy ramp than the untouched bunches, leading to on average $12 \%$ lower bunch intensity. The vertically blown-up bunches did not experience any significant intensity loss. This also had an adverse effect on the emittance ratio of the blown-up bunches to the untouched bunches, as evidenced by Fig. 10(b).

\section{B. Data analysis}

The measured waveform in one of the recorded events is shown in Fig. 11. The signal from one bunch is not fully depleted from the dBLM before the losses of the next bunch appear, leading to a certain overlap. This overlap was corrected for by fitting exponential decays to the falling edge of each individual bunch, and extrapolating down to zero. The bunches were then numerically integrated while
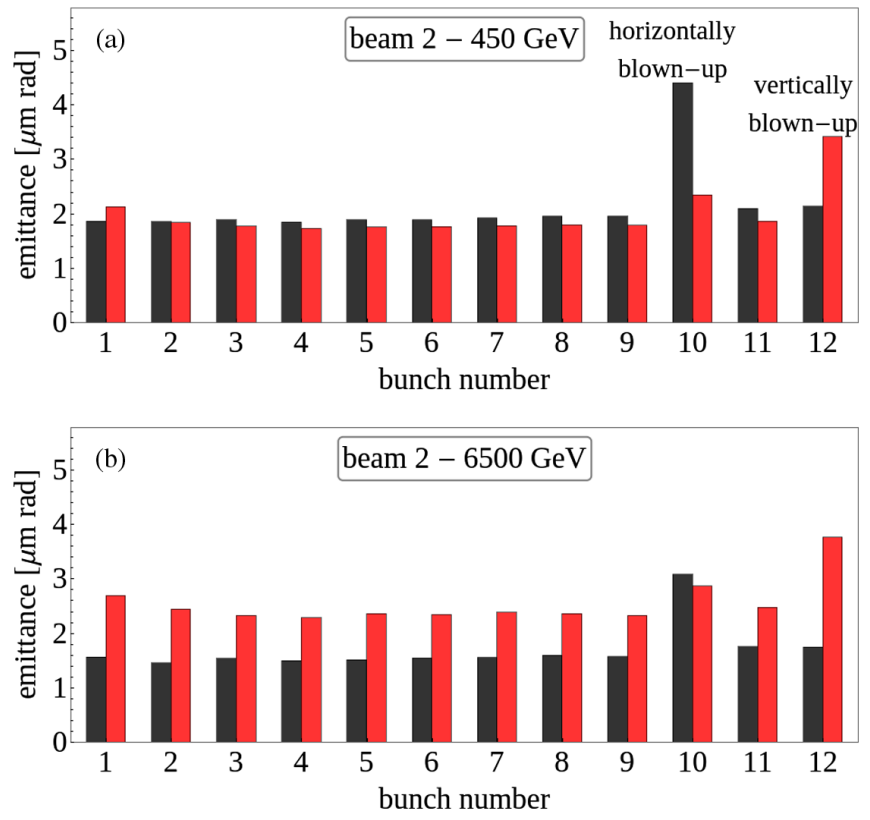

FIG. 10. Measured horizontal (black) and vertical (red) emittances of the first 12 bunches in beam 2, (a) at injection energy directly after setup and (b) at top energy after beams started colliding. 


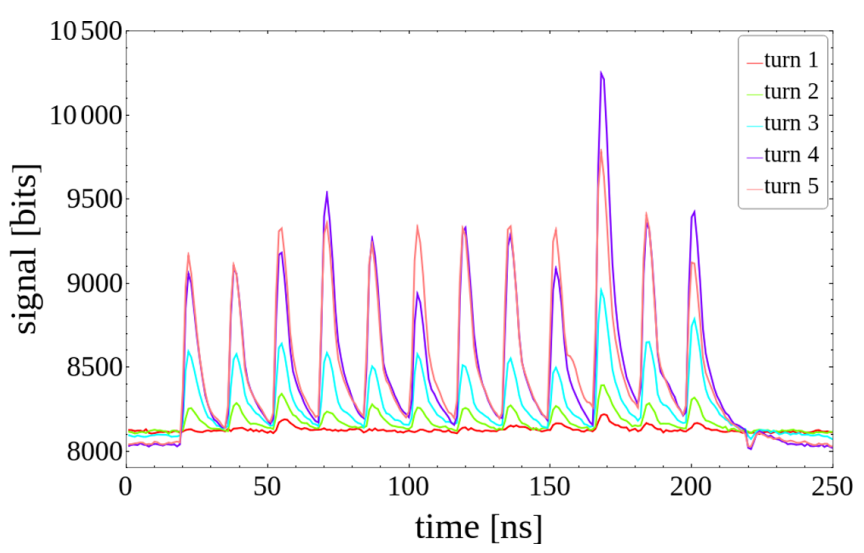

FIG. 11. The measured waveform of the 12 bunch train on a few turns surrounding the peak of the signal.

removing the overlap from preceding bunches and including the falling edge going into the following bunches.

All bunch-by-bunch measurements were normalized to their respective bunch intensities. As the variation of the bunch sizes of the nonblown up bunches was smaller than $3 \%$, their average size was considered in the analysis.

\section{Loss fluctuations and delayed losses}

As can be seen in the waveform in Fig. 11, there is a significant signal fluctuation between bunches, despite the bunches having similar parameters. This fluctuation is used for the error estimate when applying the ratio of bunches method.

Figure 12 shows an integration of the complete dBLM signal in bins of $80 \mu \mathrm{s}$ and compares it with the local ICBLM measurement. Both signals are scaled to the same peak value. The long falling edge is due to delayed losses, that is, losses being captured by the collimators over multiple turns, following the betatron oscillations. Since

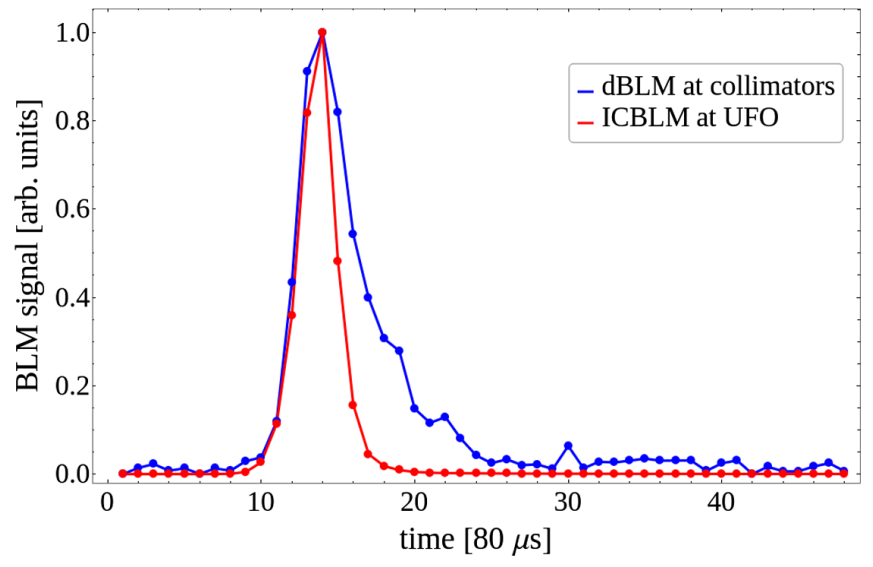

FIG. 12. Comparison of the dBLM measurement, integrated in $80 \mu \mathrm{s}$ bins, and the local ICBLM measurement. It shows that the rising edge agrees well, but that the dBLM signal has a slower falling edge due to delayed losses in the collimators. delayed losses are proportional to the measured losses on the preceding turns, the rising part agrees between the two measurements, but the falling edge is slower in the dBLM measurement. For this reason, only the rising part, up to the turn of peak losses, is used in the analysis.

\section{RESULTS}

Out of the events detected by the dBLMs, one was of significantly better quality, with sufficient length and signal-to-noise ratio to perform a detailed analysis of the dynamics of the dust particle. In this event, shown in Fig. 13, the horizontally blown-up bunch shows a significantly larger signal than the other bunches. This preference for the horizontally blown-up bunch, of about a factor of two, is seen consistently throughout the event, meaning that the dust particle was offset horizontally from the center of the beam. This event is analyzed in detail in two ways, one using the simulation tool explained in Sec. VI, and one using the ratio of bunches method discussed in Sec. VIII.

\section{A. Dust particle positions}

The result of the ratio of bunches method is shown, for the rising part of the signal, in Fig. 14. The background of the plot shows the reference bunch distribution, with the white dashed ellipses showing the 1,2, 3, and $4 \sigma$ lines. The cyan line shows the contour where the horizontal and the vertical bunch densities are equal, meaning that a dust particle on this line should give equal beam losses from the blown-up bunches.

The filled ellipses show $90 \%$ confidence regions for the estimated dust particle position, with turn 4 corresponding to the turn with peak losses. For comparison with the simulations, the estimated positions are placed in the first quadrant, but due to the symmetry of the method, any quadrant is equally possible. A typical simulated trajectory

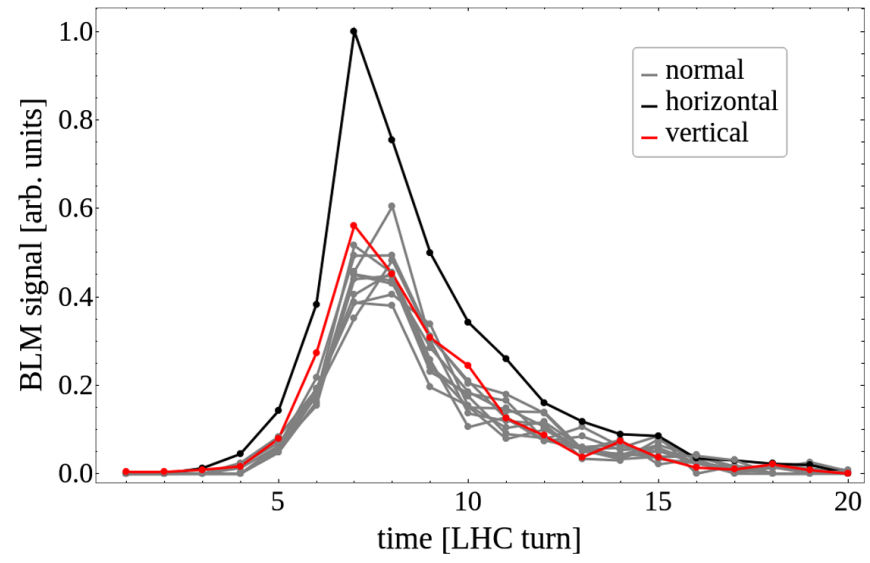

FIG. 13. Integrated signal for the 12 bunch train, each bunch individually. The horizontally blown-up bunch (black) shows a significantly larger signal throughout, and is also detected earlier than the other bunches. 


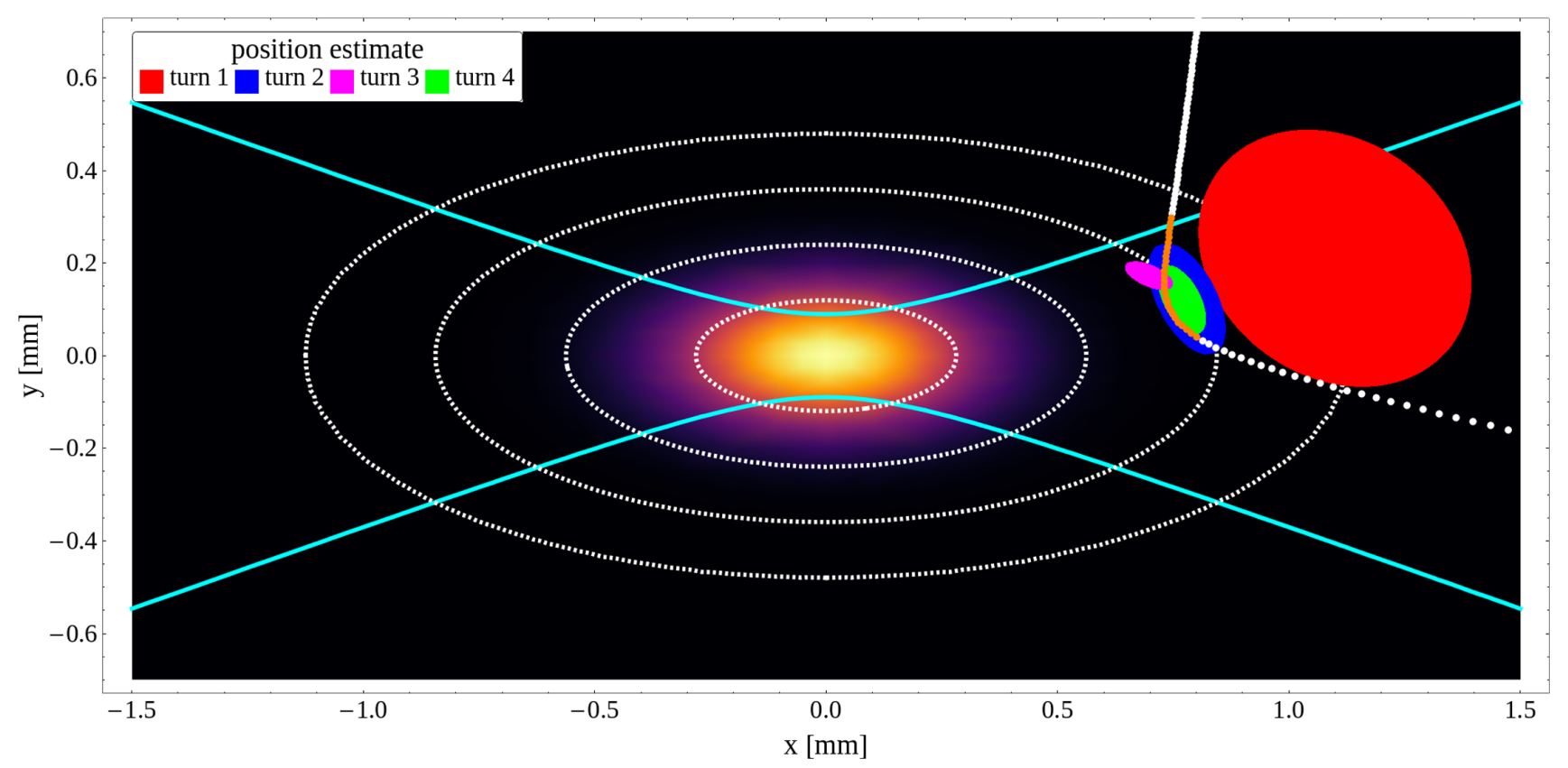

FIG. 14. Trajectory of the best fitting event from the simulations, compared to the estimated UFO position on a turn-by-turn basis.

is shown as white dots. The orange part indicates the four turns up to and including the peak of the simulated losses. The simulated trajectory corresponds to the best fit to the local ICBLM measurement that could be found using the Monte-Carlo simulations with input parameters as summarized in Table II. The reason for using the local ICBLM is their superior precision and lack of delayed losses as compared to the dBLMs. A comparison of the ICBLM measurement with the simulated losses is shown in Fig. 15, and the best fit was determined by minimizing the errors in amplitude, skewness and duration.

The estimated positions from the measurements show that it is unlikely that the dust particle was in the region where the vertically blown-up bunch should have provided more losses, for each of the four turns. This, together with the fact that the losses were rising, meaning

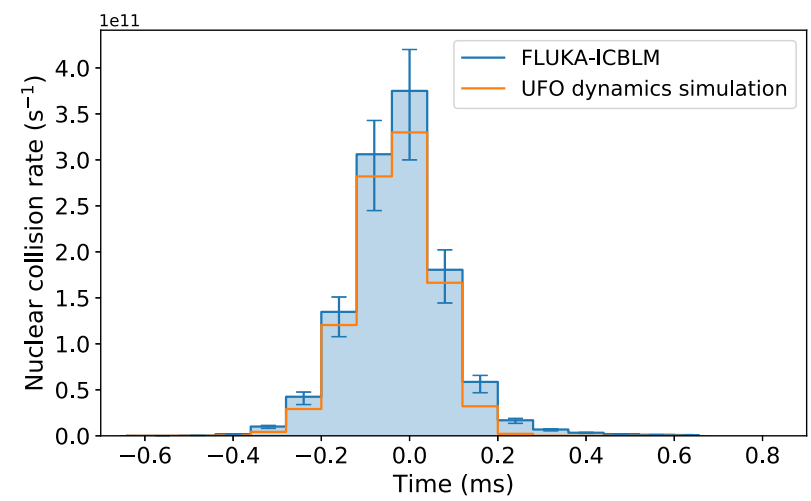

FIG. 15. Comparison between the measured beam losses by the local ICBLM and the simulated losses showing the best agreement. The total number of inelastic collisions was $5.2 \times 10^{7}$. that the dust particle penetrated deeper into the beam, is not consistent with a dust particle simply falling by the act of gravity. This supports the conclusion from the risetime analysis of the ICBLMs presented in Sec. VII, that the dust particles must have an initial negative charge, or by some other means receive a large speed before their interaction with the beam.

The position estimate for turns 2 to 4 are grouped closely together, and cannot be distinguished from each other with the given precision. However, turn 1 lies further out, showing that there is indeed a horizontal movement. These four turns are consistent with the qualitative understanding of UFO dynamics. The dust particle has a large speed just as it enters the beam, and it is quickly slowed down due to the increasing proton density once it reaches a certain depth into the beam, because it acquires a positive charge. Since it is slowed down, the dust particle positions around the peak of the losses will be close to each other, whereas the turn-byturn movement of the dust is larger as it gets further away from the turn with the peak losses.

Comparing the simulated trajectory with the measurements, there is a good agreement on the three turns where the signal was the highest, and consequently the precision and the accuracy of the position estimate the best. It should be noted that the simulations are only based on the local ICBLM measurements, yet find a good agreement with the measurements based on bunchby-bunch losses from the dBLMs. The dBLM measurements provide a strong support for the validity of the simulation tool, and additional experiments with more blown-up bunches, providing a better precision, could strengthen this further. 


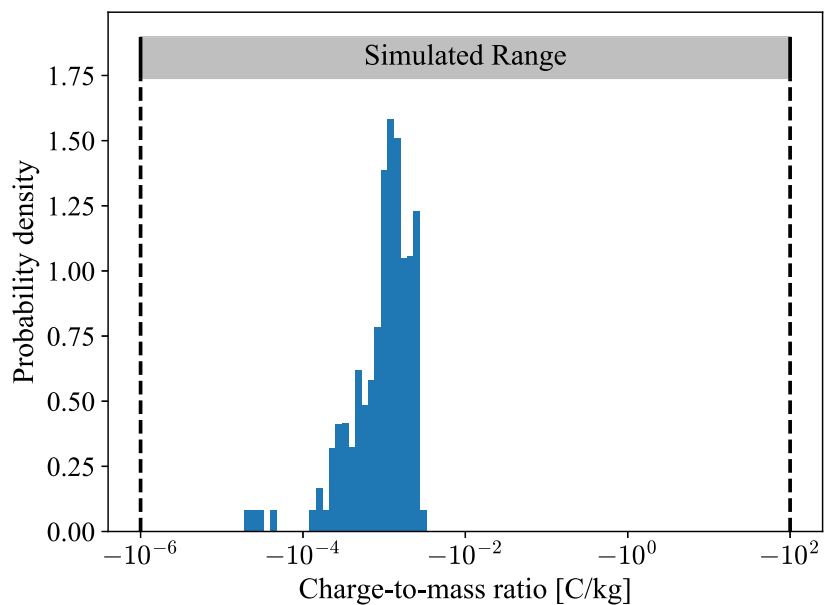

FIG. 16. Histogram showing the most likely charge-to-mass ratios as determined from the $\mathrm{MC}$ simulations. A concentration is found around $-1.20 \times 10^{-3} \mathrm{C} / \mathrm{kg}$ for this particular event.

\section{B. Initial conditions}

From the Monte-Carlo simulations it is also possible to estimate the most likely initial conditions of the dust particle in this particular event. In Fig. 16, a histogram of the charge-to-mass ratios used in the MC simulation is shown. An error of 5\% is allowed for the shape parameters, width $\omega$ and skewness $\alpha$, when comparing the beam loss time profiles of the simulations with the measurement. This provides a distribution of the best fitting simulations. They show a concentration around a charge-to-mass ratio of $-1.20 \times 10^{-3} \mathrm{C} / \mathrm{kg}$. In particular, no simulated events starting with a neutral charge-to-mass ratio were able to reproduce the measured beam losses.

A similar histogram over the starting horizontal position is shown in Fig. 17. It shows a concentration around $0.87 \mathrm{~mm}$. There is nothing in the structure of the beam

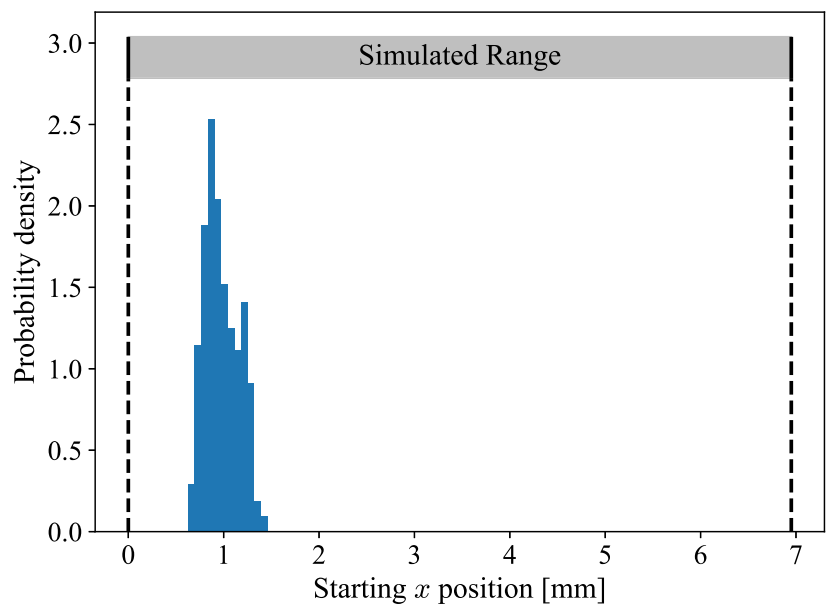

FIG. 17. Histogram showing the most likely horizontal starting positions as determined from the MC simulations. A concentration is found around $0.87 \mathrm{~mm}$, for this particular UFO event.

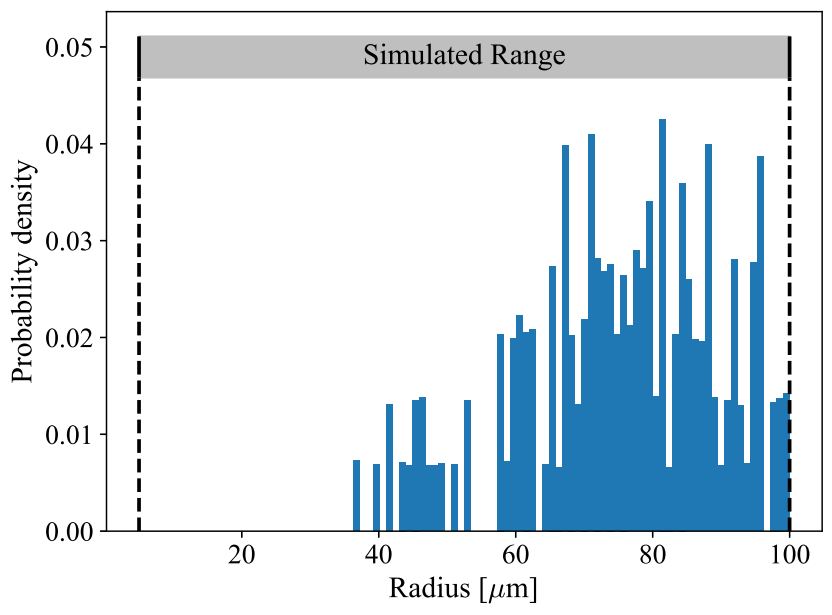

FIG. 18. Histogram showing the most likely dust particle radii as determined from the MC simulations. The average is $81.5 \mu \mathrm{m}$, for this particular UFO event.

screen to explain why this particular offset could be interesting, however this set of starting positions appears to be the most probable values for this event.

The dust particle radii are shown in Fig. 18. They show a relatively large spread, but with a minimum radius of about $40 \mu \mathrm{m}$. Dust particles with a larger radius are heavier and tend to go more deeply into the beam before being ejected.

\section{CONCLUSION}

In this paper, the different types of UFOs that have been observed in the LHC were introduced and discussed. UFOs are believed to consist of dust particles that enter the beam, causing beam losses which can e.g., trigger magnet quenches and negatively impact machine availability. While the MKI, ULO and 16L2 type UFOs have been explained and mitigated, the issue with spurious UFOs distributed throughout the accelerator remains, and in particular their source and trigger mechanism remains unknown.

A number of advances on the theoretical aspect have been presented. The UFO charging rate due to the beamdust interaction, necessary for correct modeling of the UFO dynamics, was updated and validated against FLUKA simulations, showing a good agreement. The dynamics simulation tool was further validated against both ICBLM and dBLM measurements. It can correctly reproduce the ICBLM measurements where the beam loss time profiles are negatively skewed, including rise times, absolute signal amplitudes, and decay times. However, events with positively skewed time profiles, accounting for roughly half of all recordings, are to be understood.

The ratio of losses method for measuring the UFO dust particle position in relation to the beam center was explored during normal beam operation. This method uses the 
dBLMs, having an individual bunch resolution, and a set of two blown-up bunches per beam with a larger beam size in one plane each, compared to the reference bunches. A good agreement was found for the UFO penetration depth into the beam, comparing the measured position with the UFO dynamics simulation tool. This provides a confirmation of the validity of the simulation tool.

The dBLM measurements additionally indicate that the dust particle had a horizontal component to its velocity while approaching the beam in at least one event. Furthermore, the Monte-Carlo simulations looking at loss rise times cannot explain the majority of the events if the dust particles are initially neutral. It was found that a charge-to-mass ratio of $-5 \times 10^{-3} \mathrm{C} / \mathrm{kg}$ or smaller produces a comparable distribution of rise times. Consequently there is a strong indication that the dust particle is initially charged, or that it by some other means acquires a large speed during its approach to the beam not explainable by gravity. This has an important effect on the UFO dynamics and must be considered when estimating their impact on machine availability in future LHC operation, as well as for other accelerators with positively charged beams.

\section{ACKNOWLEDGMENTS}

This work was supported by the High Luminosity Large Hadron Collider project.

[1] T. Baer, A. Cerutti, N. Ferrari, B. Garrel, E. Goddard, S. Holzer, A. Jackson, V. Lechner, M. Mertens, E. Misiowiec, D. Nebot, A. Busto, J. Nordt, V. Uythoven, J. Vlachoudis, C. Wenninger, F. Zamantzas, N. Fuster, M. Barnes, and F. Zimmerman, UFOs in the LHC: Observations, studies and extrapolations, in Proc. 3rd Int. Particle Acc. Conf. (JACoW, Geneva, Switzerland, 2012).

[2] S. Rowan, LHC main dipole magnet circuits: Sustaining near-nominal beam energies, Ph.D. thesis, University of Glasgow, 2016.

[3] T. Baer et al., UFOs in the LHC after LS1, Proceedings Chamonix 2012 Workshop on LHC Performance (CERN, Geneva, Switzerland, 2012).

[4] D. Mirarchi, G. Arduini, M. Giovannozzi, A. Lechner, S. Redaelli, and J. Wenninger, Special Losses During LHC Run 2, Paper presented at the 9th LHC operations Evian workshop, Evian, France (CERN, Geneva, Switzerland, 2019).

[5] F. Zimmermann, J. T. Seeman, M. Zolotorev, and W. Stoeffl, Trapped macroparticles in electron storage rings, in Proceedings of the Particle Accelerator Conference, Dallas, TX, 1995 (IEEE, New York, 1995), Vol. 1, pp. 517-519.

[6] D. Sagan, Mass and charge measurement of trapped dust in the CESR storage ring, Nucl. Instrum. Methods Phys. Res., Sect. A 330, 371 (1993).

[7] H. Saeki, T. Momose, and H. Ishimaru, Observations of dust trapping phenomena in the TRISTAN accumulation ring and a study of dust removal in a beam chamber, Rev. Sci. Instrum. 62, 874 (1991).
[8] Y. Tanimoto, T. Honda, and S. Sakanaka, Experimental demonstration and visual observation of dust trapping in an electron storage ring, Phys. Rev. Accel. Beams 12, 110702 (2009).

[9] R. Takai, T. Honda, T. Nogami, T. Obina, Y. Tanimoto, and M. Tobiyama, A New Stripline Kicker for PF-AR Transverse Feedback Damper, in 5th International Beam Instrumentation Conference (2017), p. TUPG13, https:// doi.org/10.18429/JACoW-IBIC2016-TUPG13.

[10] S. Terui et al., Observation of Pressure Bursts in the SuperKEKB Positron Ring, in Proc. 9th International Particle Accelerator Conference (IPAC'18), Vancouver, BC, Canada, April 29-May 4, 2018, International Particle Accelerator Conference No. 9 (JACoW Publishing, Geneva, Switzerland, 2018), pp. 2830-2832, https:// doi.org/10.18429/JACoW-IPAC2018-WEPML058.

[11] S. Rowan, A. Apollonio, B. Auchmann, A. Lechner, O. Picha, W. Riegler, H. Schindler, R. Schmidt, and F. Zimmermann, Interactions between macroparticles and high-energy proton beams, in 6th International Particle Accelerator Conference, IPAC 2015, 2 (JACoW, Geneva, Switzerland, 2015), pp. 2112-2115.

[12] B. Goddard, P. Adraktas, T. Baer, M. J. Barnes, F. Cerutti, A. Ferrari, N. Garrel, A. Gerardin, M. Guinchard, A. Lechner, A. Masi, V. Mertens, R. M. Ballester, S. Redaelli, J. Uythoven, V. Vlachoudis, and F. Zimmermann, Transient beam losses in the LHC injection kickers from micron scale dust particles, in Proceedings of the 3rd International Particle Accelerator Conference, New Orleans, LA, 2012 (IEEE, Piscataway, NJ, 2012), p. 2044.

[13] T. Baer, Very fast losses of the circulating LHC beam, their mitigation and machine protection, Ph.D. thesis, Universität Hamburg, 2013.

[14] T. Baer, M. Barnes, W. Bartmann, C. Bracco, E. Carlier, C. Chanavat, N. Drosdal, N. Garrel, B. Goddard, V. Kain, V. Mertens, J. Uythoven, J. Wenninger, and M. Zerlauth, MKI UFOs at injection, CERN Tech. Report No. CERN-ATSNote-2011-065 MD, 2011.

[15] T. Baer, M. Barnes, W. Bartmann, C. Bracco, E. Carlier, B. Dehning, N. Garrel, B. Goddard, S. Jackson, M. Jimenez, V. Kain, V. Mertens, M. Misiowiec, A. Nordt, G. Papotti, J. Uythoven, J. Wenninger, M. Zerlauth, C. Zamantzas, and F. Zimmermann, MD on UFOs at MKIs and MKQs, CERN Tech. Report No. CERN-ATS-Note-2012-018 MD, 2012.

[16] T. Böhlen, F. Cerutti, M. Chin, A. Fassò, A. Ferrari, P. Ortega, A. Mairani, P. Sala, G. Smirnov, and V. Vlachoudis, The FLUKA Code: Developments and Challenges for High Energy and Medical Applications, Nucl. Data Sheets 120, 211 (2014).

[17] G. Battistoni, T. Boehlen, F. Cerutti, P. W. Chin, L. S. Esposito, A. Fassò, A. Ferrari, A. Lechner, A. Empl, A. Mairani, A. Mereghetti, P. G. Ortega, J. Ranft, S. Roesler, P. R. Sala, V. Vlachoudis, and G. Smirnov, Overview of the FLUKA code, Annals of Nuclear Energy 82, 10 (2015), joint International Conference on Supercomputing in Nuclear Applications and Monte Carlo 2013, SNA + MC 2013. Pluri- and Trans-disciplinarity, Towards New Modeling and Numerical Simulation Paradigms.

[18] FLUKA website (2020), https://fluka.cern. 
[19] G. Kotzian, M. Barnes, L. Ducimetiere, B. Goddard, and W. Hfle, Emittance Growth at LHC Injection from SPS and LHC Kicker Ripple, in Proc. 11th European Particle Accelerator Conference (EPAC08), Genoa, Italy, June 23-27 (EPS-AG, Genoa, Italy, 2008).

[20] D. Mirarchi, R. Bruce, M. Giovannozzi, P. Hermes, S. Redaelli, B. Salvachua, G. Valentino, and J. Wenninger, LHC aperture and ULO restrictions: are they a possible limitation in 2016?, CERN-ACC-2015-376, Proceedings of the 6th Evian Workshop, Evian, France (CERN, Geneva, Switzerland, 2015).

[21] A. Lechner et al., Beam Loss Measurements for Recurring Fast Loss Events During 2017 LHC Operation Possibly Caused by Macroparticles, in Proc. 9th International Particle Accelerator Conference (IPAC'18), Vancouver, BC, Canada, April 29-May 4, 2018, International Particle Accelerator Conference No. 9 (JACoW Publishing, Geneva, Switzerland, 2018), pp. 780-783, https://doi.org/ 10.18429/JACoW-IPAC2018-TUPAF040.

[22] O. Bruning (The LHC Collaboration), LHC design report. Vol. 1. Vol. 1 (CERN, Geneva, 2004) Chap. 18.

[23] J. Jimenez et al., Observations, analysis and mitigation of recurrent LHC beam dumps caused by fast losses in arc half-cell 16L2, 9th International Particle Accelerator Conference (JACoW, Geneva, Switzerland, 2018).

[24] L. Mether, D. Amorim, G. Arduini, X. Buffat, G. Iadarola, A. Lechner, E. Métral, D. Mirarchi, G. Rumolo, and B. Salvant, 16L2: Operation, Observations and Physics Aspects, Paper presented at the 8th LHC operations Evian workshop, Evian, France (CERN, Geneva, Switzerland, 2018).

[25] N. F. Martinez, F. Zimmermann, T. Baer, M. Giovannozzi, E. B. Holzer, E. Nebot, A. Nordt, M. Sapinski, and Z. Yang, Simulation studies of macroparticles falling into the LHC proton beam, in Proceedings of the 2nd International Particle Accelerator Conference, San Sebastiáán, Spain (EPS-AG, Spain, 2011), p. 634.

[26] L. K. Grob, C. Neves, A. Apollonio, J. D. Busom, C. Charvet, A. T. P. Fontenla, E. G.-T. Valdivieso, H. Kos, and R. Schmidt, Dust analysis from LHC vacuum system to identify the source of macro particle-beam-interactions, in Proceedings of IPAC2019 (JACoW, Geneva, Switzerland, 2019), p. 1082.

[27] E. Ciapala, F. Rodríguez-Mateos, R. Schmidt, and J. Wenninger, The LHC post-mortem system, CERN Tech. Report No. LHC-PROJECT-NOTE-303, 2002.

[28] B. Dehning, E. Effinger, J. Emery, G. Ferioli, G. Guaglio, E. B. Holzer, D. Kramer, L. Ponce, V. Prieto, M. Stockner, and C. Zamantzas, The LHC beam loss measurement system, in Proceedings of the 22nd Particle Accelerator Conference, PAC-2007, Albuquerque, NM (IEEE, New York, 2007), p. 4192.

[29] M. Stockner, B. Dehning, C. Fabjan, G. Ferioli, and E. B. Holzer, Measurements and simulations of ionization chamber signals in mixed radiation fields for the LHC BLM system, in 2006 IEEE Nuclear Science Symposium Conference Record, Vol. 3 (2006), pp. 1342-1345, https:// doi.org/10.1109/NSSMIC.2006.354151.

[30] D. Kramer, Design and implementation of a detector for high flux mixed radiation fields, Ph.D. thesis, Technical
University of Liberec, 2008, http://cds.cern.ch/record/ 1139227/files/CERN-THESIS-2008-090.pdf.

[31] A. Boccardi, M. B. Marin, T. Levens, B. Szuk, W. Vigan, and C. Zamantzas, A Modular Approach to Acquisition Systems for Future CERN Beam Instrumentation Developments, in 15th International Conference on Accelerator and Large Experimental Physics Control Systems (2015), p. THHB2O02, https://doi.org/10.18429/JACoW-ICALEPCS2015-THHB2O02.

[32] A. Gorzawski, S. Redaelli, N. F. Martinez, H. G. Morales, A. Mereghetti, X. Cai, G. Valentino, and R. B. Appleby, Fast loss analysis with LHC diamond detectors in 2017, CERN Tech. Report No. CERN-ACC-NOTE-2018-0041, 2018.

[33] A. Lechner, B. Auchmann, T. Baer, C. B. Castro, R. Bruce, F. Cerutti, L. S. Esposito, A. Ferrari, J. M. Jowett, A. Mereghetti, F. Pietropaolo, S. Redaelli, B. Salvachua, M. Sapinski, M. Schaumann, N. V. Shetty, and V. Vlachoudis, Validation of energy deposition simulations for proton and heavy ion losses in the CERN Large Hadron Collider, Phys. Rev. Accel. Beams 22, 071003 (2019).

[34] F. Zimmermann, M. Giovannozzi, and A. Xagkoni, Interaction of macro-particles with LHC proton beam, in Proceedings of the International Particle Accelerator Conference, Kyoto, Japan (ICR, Kyoto, 2010), p. 492.

[35] P. Bélanger, Generalizing the method of images for complex boundary conditions: Application on the LHC beam screen, arXiv:1905.03405.

[36] Tanabashi (Particle Data Group), Particle Data Guide. 33. Passage of particles through matter, Phys. Rev. D 98, 030001 (2018).

[37] E. J. Kobetich and R. Katz, Energy deposition by electron beams and $\delta$ rays, Phys. Rev. 170, 391 (1968).

[38] D. A. Mendis, Progress in the study of dusty plasmas, Plasma Sources Sci. Technol. 11, A219 (2002).

[39] B. Lindstrom, A. Apollonio, P. Bélanger, M. Dziadosz, A. Gorzawski, L. Grob, E. B. Holzer, A. Lechner, R. Schmidt, M. Valette, D. Valuch, and D. Wollmann, Results of UFO dynamics studies with beam in the LHC, J. Phys. Conf. Ser. 1067, 022001 (2018).

[40] A. Gorzawski, R. B. Appleby, M. Giovannozzi, A. Mereghetti, D. Mirarchi, S. Redaelli, B. Salvachua, G. Stancari, G. Valentino, and J. F. Wagner, Probing LHC halo dynamics using collimator loss rates at 6.5 TeV, Phys. Rev. Accel. Beams 23, 044802 (2020).

[41] (The CMS Collaboration), CMS Physics Analysis Summary, CERN Tech. Report No. CMS-PAS-EWK-11-001, 2011.

[42] T. Mitsuhashi, E. Bravin, F. Roncarolo, and G. Trad, First observation of the LHC beam halo using a synchrotron radiation coronagraph, in 8th International Particle Accelerator Conference (JACoW, Geneva, 2017), p. TUOAB2.

[43] T. Lefevre, G. Burtin, E. Bravin, A. Goldblatt, A. Guerrero, A. Jeff, F. Roncarolo, and A.S. Fisher, First beam measurements with the LHC synchrotron light monitors, Proceedings of the 1st Int. Particle Accelerator Conf. IPAC2010, 3 (2010), https://accelconf.web.cern .ch/IPAC10/papers/mope057.pdf. 